\title{
Circulating endothelial microparticles: a promising biomarker of acute kidney injury after cardiac surgery with cardiopulmonary bypass
}

\author{
Jian Ma ${ }^{1,2,3,4 \#}$, Hao-Xiang Yuan ${ }^{1,2,3,4 \pi} \wedge$, Ya-Ting Chen ${ }^{1,2,3,4 \#}$, Da-Sheng Ning ${ }^{1,2,3,4} \wedge$, Xiao-Jun Liu ${ }^{1,2,3,4} \wedge$, \\ Yue-Ming Peng ${ }^{1,2,3,4} \wedge$, Chao Chen ${ }^{1,2,3,4} \wedge$, Yuan-Kai Song ${ }^{1,2,3,4}$, Yu-Peng Jian ${ }^{1,2,3,4} \wedge$, Yan Li $^{1,2,3,4} \wedge$, \\ Zui Liu ${ }^{1,2,3,4}$, Zhi-Jun Ou ${ }^{2,3,4,5}$, Jing-Song Ou ${ }^{1,2,3,4,6 \wedge}$
}

${ }^{1}$ Division of Cardiac Surgery, Heart Center, The First Affiliated Hospital, Sun Yat-sen University, Guangzhou, China; ${ }^{2}$ National-Guangdong Joint Engineering Laboratory for Diagnosis and Treatment of Vascular Diseases, Guangzhou, China; ${ }^{3}$ NHC key Laboratory of Assisted Circulation (Sun Yat-sen University), Guangzhou, China; ${ }^{4}$ Guangdong Provincial Engineering and Technology Center for Diagnosis and Treatment of Vascular Diseases, Guangzhou, China; ${ }^{5}$ Division of Hypertension and Vascular Diseases, Heart Center, The First Affiliated Hospital, Sun Yat-sen University, Guangzhou, China; ${ }^{6}$ Guangdong Provincial Key Laboratory of Brain Function and Disease, Guangzhou, China

Contributions: (I) Conception and design: JS Ou; (II) Administrative support: JS Ou; (III) Provision of study materials or patients: JS Ou; (IV) Collection and assembly of data: J Ma, HX Yuan, YT Chen, DS Ning, XJ Liu, C Chen, YK Song, Y Li, Z Liu, ZJ Ou; (V) Data analysis and interpretation: JS Ou, J Ma, HX Yuan, YT Chen, YM Peng, YP Jian; (VI) Manuscript writing: All authors; (VII) Final approval of manuscript: All authors.

\#These authors contribute equally to this work.

Correspondence to: Jing-Song Ou, MD, PhD. Division of Cardiac Surgery, The First Affiliated Hospital, Sun Yat-sen University, 58 Zhong Shan Er Road, Guangzhou 510080, China. Email: oujs@mail.sysu.edu.cn; oujs2000@163.com.

Background: Current diagnostic strategies for acute kidney injury (AKI) after cardiac surgery with cardiopulmonary bypass (CPB) are nonspecific and limited. Previously, we demonstrated that circulating microparticles (MPs) in patients with valve heart disease (VHD) and congenital heart diseases (CHD) induce endothelial dysfunction and neutrophil chemotaxis, which may result in kidney injury. We also found that circulating MPs increase after cardiac surgery with CPB and are related to cardiac function. However, the relationship between circulating MPs and AKI after CPB is unknown.

Methods: Eighty-five patients undergoing cardiac surgery with CPB were enrolled. Patients were divided into AKI and non-AKI groups based on the serum creatinine levels at $12 \mathrm{~h}$ and $3 \mathrm{~d}$ post-CPB. Circulating MPs were isolated from plasma, and their levels including its subtypes were detected by flow cytometer. Independent risk factors for the CPB-associated AKI (CPB-AKI) were determined by multivariate logistic regression analysis. Receiver operating characteristic (ROC) analysis was used to measure the prognostic potential of CPB-AKI.

Results: The morbidity of AKI at $12 \mathrm{~h}$ and $3 \mathrm{~d}$ after cardiac surgery with CPB was $40 \%$ and $31.76 \%$, respectively. The concentrations of total MPs and platelet-derived MPs (PMP) remained unchanged at $12 \mathrm{~h}$ and then increased at $3 \mathrm{~d}$ post-CPB, while that of endothelial-derived MPs (EMP) increased at both time points. In patients with AKI, PMP and EMP were elevated compared with the patients without AKI. However, no significant change was detected on monocyte-derived MPs (MMP) at $12 \mathrm{~h}$ and $3 \mathrm{~d}$ post-CPB. The logistic regression analysis showed that EMP was the independent risk factor for AKI both at $12 \mathrm{~h}$ and $3 \mathrm{~d}$ post-CPB. The area under ROC for the concentrations of EMP at $12 \mathrm{~h}$ and $3 \mathrm{~d}$ post-CPB was 0.86 and

^ ORCID: Jian Ma, 0000-0003-3394-5214; Hao-Xiang Yuan, 0000-0003-2886-9878; Ya-Ting Chen, 0000-0003-2534-3643; Da-Sheng Ning, 00000002-6355-4794; Xiao-Jun Liu, 0000-0003-2161-9622; Yue-Ming Peng, 0000-0002-2966-974X; Chao Chen, 0000-0002-4177-3275; Yuan-Kai Song, 0000-0002-7336-5110; Yu-Peng Jian, 0000-0002-5952-7769; Yan Li, 0000-0003-2450-3836; Zui Liu, 0000-0002-8720-6954; Jing-Song Ou, 0000$0001-5517-3142$. 
0.91 , with the specificity up to 0.88 and 0.91 , respectively.

Conclusions: Circulating EMP may serve as a potential biomarker of AKI after cardiac surgery with CPB.

Keywords: Circulating microparticles; endothelial-derived microparticles (EMP); cardiopulmonary bypass (CPB); cardiac surgery; acute kidney injury (AKI)

Submitted Dec 07, 2020. Accepted for publication Feb 22, 2021.

doi: 10.21037/atm-20-7828

View this article at: http://dx.doi.org/10.21037/atm-20-7828

\section{Introduction}

Acute kidney injury (AKI) is a common complication in patients undergoing cardiac surgery with cardiopulmonary bypass (CPB), with morbidity up to $30 \%$ (1). CPB-associated AKI (CPB-AKI) increases the need for mechanical ventilation, days of intensive care, and mortality (2). In CPB, aorta cross-clamping and non-pulsatile blood flow contribute to the inflammatory responses and oxidative stress injury, which may induce CPB-AKI (3). The early diagnosis of $\mathrm{CPB}-\mathrm{AKI}$ is difficult, which is a challenge for the therapeutic strategy (3). Nowadays, microparticles (MPs), especially its subtype and endothelial-derived microparticles (EMP) from circulation are becoming a promising diagnostic biomarker for AKI (4).

Circulating MPs ranging from 100 to $1,000 \mathrm{~nm}$ are derived from circulating cells upon activation (5). Concentrations of MPs are closely associated with cardiovascular disease such as hypercholesterolemia, atherosclerosis, congenital heart diseases (CHD), and valve heart disease (VHD), and increase after cardiac surgery with CPB (5-10). More importantly, a nanoparticle tracking analysis reveals a higher concentration of circulating MPs in patients with acute heart failure at $12 \mathrm{~h}$ after cardiac surgery with CPB (10). Circulating MPs are distinguished based on the source cells such as platelet-derived MP (PMP), EMP, and monocyte-derived MP (MMP) $(11,12)$. Recent studies found high concentrations of MPs after CPB $(10,13,14)$. However, the relationship between CPB-AKI and circulating MPs, especially its subpopulations, remains unclear.

More evidence show that microparticles mediate inflammation, coagulation, oxidative stress injury, and endothelial dysfunction in patients with renal disease and become a promising biomarker of various renal diseases such as thrombotic microangiopathies, nephrotic syndrome, IgA nephropathy, and renal failure $(4,15)$. In view of molecular mechanism, MPs, especially EMP, induce endothelial dysfunction by increasing the release of inflammatory cytokines and reducing nitric oxide (5-8,16-18). Recently, we found that circulating MPs from patients with VHD induce chemokines expression and renal neutrophil chemotaxis, which partly contribute to renal dysfunction (18). Recent investigations on the correlation between circulating EMP and renal dysfunction found that EMP are elevated in patients with chronic kidney disease (CKD) or septic patients with AKI (19). However, the relationship between circulating EMP and $\mathrm{AKI}$ after $\mathrm{CPB}$ also remains unknown.

We hypothesized that the activation of the endothelium during CPB could lead to the release of EMP, and the elevated EMP are correlated with kidney injury and may be a diagnostic biomarker for CPB-AKI. Thus, flow cytometry was performed to analyze circulating MPs and its subpopulation including EMP, PMP, and MMP in patients undergoing cardiac surgery with $\mathrm{CPB}$ and the correlations between MPs especially EMP and AKI were analyzed.

We present the following article in accordance with the STARD reporting checklist (available at http://dx.doi. org/10.21037/atm-20-7828).

\section{Methods}

\section{Study cohort}

In this retrospective observational study, 85 adult patients, diagnosed with heart disease undergoing cardiac surgery with CPB were enrolled from the First Affiliated Hospital, Sun Yat-sen University. Patients with trauma, shock, sepsis, chronic pulmonary disease, cancer, and severe hepatic dysfunction were excluded from the study. The patients were assigned into AKI and non-AKI groups based on the serum creatinine levels at $12 \mathrm{~h}$ and $3 \mathrm{~d}$ post-CPB according to the Kidney Disease Improving Global Guidelines (KDIGO) Clinical Practice Guidelines for AKI (20). Summarily, serum creatinine increased in patients after operation by $\geq 26.5 \mu \mathrm{mol} / \mathrm{L}$ within $48 \mathrm{~h}$ or $\geq 1.5$ times 
baseline level occurred within 7 days prior to the operation are diagnosed with AKI based on KIDGO. This study complied with the declaration of Helsinki (as revised in 2013) and was also approved by the Ethics Review Board of the First Affiliated Hospital, Sun Yat-sen University [(2011)14]. All subjects recruited in this study signed the written informed consent.

\section{Collection of clinical information}

The clinical data of patients enrolled in this study including age, sex, weight, height, diabetes mellitus (DM), hypertension, cardiac disease history $(\mathrm{CDH})$, and personal history (drinking and smoking) were collected. The preoperative data such as ultrasonography of the heart, serum creatinine, uric acid, and blood routine, were collected alongside the intraoperative information: subtypes of cardiac surgery, operation time, $\mathrm{CPB}$ time and cross clamp time, and serum creatinine at the $12 \mathrm{~h}$ and $3 \mathrm{~d}$ post-CPB.

\section{MPs isolation}

MPs were isolated from enrolled patients by differential centrifugation as previously reported $(5,10,18)$. According to the methodological guidelines to study MPs (21), intravenous blood was drawn by the vacuum citrateanticoagulant tubes and the first tube was discarded to avoid the influence of blood injury induced by venipuncture. The blood was then centrifuged at 2,000 g for $20 \mathrm{~min}$ at $4{ }^{\circ} \mathrm{C}$ twice to discard the deposits of blood cells within $1 \mathrm{~h}$. The plasma was gently absorbed at $0.5 \mathrm{~cm}$ above the hemocyte layer to reduce the pollution of hemocytes. Afterwards, the platelet-rich plasma was centrifuged at $11,000 \mathrm{~g}$ at $4{ }^{\circ} \mathrm{C}$ for $2 \mathrm{~min}$ to pellet platelets. The upper platelet-poor plasma was stored at $-80^{\circ} \mathrm{C}$ before use.

\section{Flow cytometry (FCM)}

The platelet-poor plasma was thawed in the water bath at $37^{\circ} \mathrm{C}$ and kept at room temperature (RT) before detection. The measurement of MPs and its subpopulations was done as previously done by FCM (18). The plasma was incubated with AnnexinV-FITC with binding buffer for $30 \mathrm{~min}$ in the dark at RT to stain the total MPs. EMP, PMP, and MMP were labeled with specific biomarkers of their source cell such as CD144 for endothelial cells, CD41 for platelets and CD14 for monocytes, respectively. The plasma was then divided into three samples and incubated with CD144 conjugated with $\mathrm{PE}, \mathrm{CD} 41$ conjugated with percpcy5.5 and CD14 conjugated with APC, individually. The plasma was incubated with a final volume of $300 \mu \mathrm{L}$ at RT for $30 \mathrm{~min}$. To quantity the MPs, the Megmix-Plus SSC beads with four diameters $(160,200,240$, and $500 \mathrm{~nm})$ were performed to set MPs gate according to the instruction. The fluorescent microspheres with $3.8 \mu \mathrm{m}$ in diameter were added before being detected by the high-sensitivity flow cytometer (Cytoflex S, Beckman coulter).

\section{Statistical analyses}

Continuous variables were described as mean \pm standard deviation (mean $\pm \mathrm{SD}$ ) unless specially illustrated and the categorical variables were represented as frequency $(\mathrm{N})$. For our risk factor analysis, missing values were stochastically imputed using a multiple imputation method. The comparison of concentrations of MPs and its subpopulations were performed with the Welch's test. The other continuous variables were compared with unpaired $t$-test. Categorical variables were compared using $\chi^{2}$ analysis.

Covariant screening was used to screen for possible confounders. The screening criteria included factors that changes $>$ by $10 \%$ when covariates are introduced into the underlying model or when covariates are removed from the regression model. Univariate analysis was conducted for the potential risk factors of AKI. A systemic search was conducted to exclude highly correlated risk factors. If two variables had a high Pearson correlation, the one with the largest average absolute correlation was removed from consideration. After univariate analysis, the significant factors were introduced into the multivariate logistic analysis to find out the independent risk factors.

The receiver operating characteristic curve (ROC) and area under curve (AUC) were calculated to evaluate the diagnostic value of the promising biomarkers for AKI. AUC confidence interval and significance test were calculated using nonparametric repeated sampling method (Bootstrap resampling times $=500$ ).

The statistical software SPSS 17.0 and R3.6.3 was used for data analysis, and $\mathrm{P}<0.05$ was considered statistically significant.

\section{Results}

\section{Demographic and perioperative clinical characteristics}

The morbidity of AKI at $12 \mathrm{~h}(12 \mathrm{~h}$ post-CPB) and $3 \mathrm{~d}$ (3 d post-CPB) after cardiac surgery with $\mathrm{CPB}$ was $40 \%$ 
Table 1 Demographic and perioperative characteristics between AKI and Non-AKI group at $12 \mathrm{~h}$ and $3 \mathrm{~d}$ post-CPB

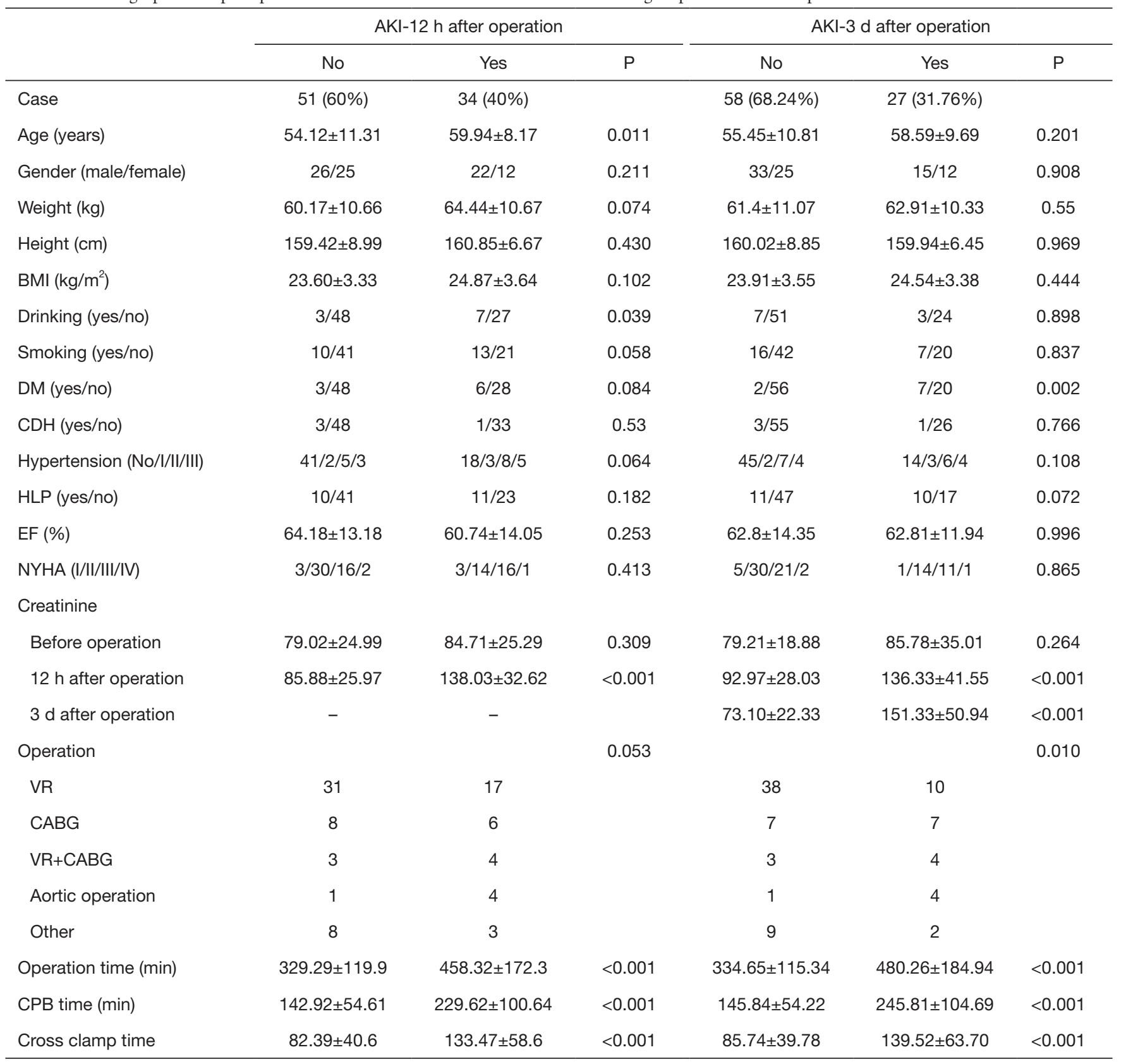

Data are presented as mean \pm SD or number of patients (\%). AKI, acute kidney injury; CPB, cardiopulmonary bypass; BMI, body mass index; EF, ejection fraction; DM, diabetes mellitus; CDH, cardiac disease history; HLP, hyperlipoidemia; NYHA, New York Heart Association; VR, valve replacement /repair; CABG, coronary artery bypass grafting.

and $31.76 \%$ respectively as described in Table 1 . The perioperative characteristics including gender, weight, height, BMI, smoking, $\mathrm{CDH}$, hypertension, hyperlipidemia (HLP), ejection fraction (EF)\% and the grade of New York Heart Association (NYHA) and the levels of creatinine before surgery were not significantly changed between
non-AKI and AKI groups at $12 \mathrm{~h}$ post-CPB or $3 \mathrm{~d}$ postCPB (Table 1). The percentages of old age and history of drinking were higher in AKI at $12 \mathrm{~h}$ post-CPB, but not at $3 \mathrm{~d}$ post-CPB (Table 1). Compared with non-AKI group, the underlying disease $\mathrm{DM}$ was found in the patients with AKI at $3 \mathrm{~d}$ post-CPB. The occurrence of AKI at $12 \mathrm{~h}$ post-CPB 

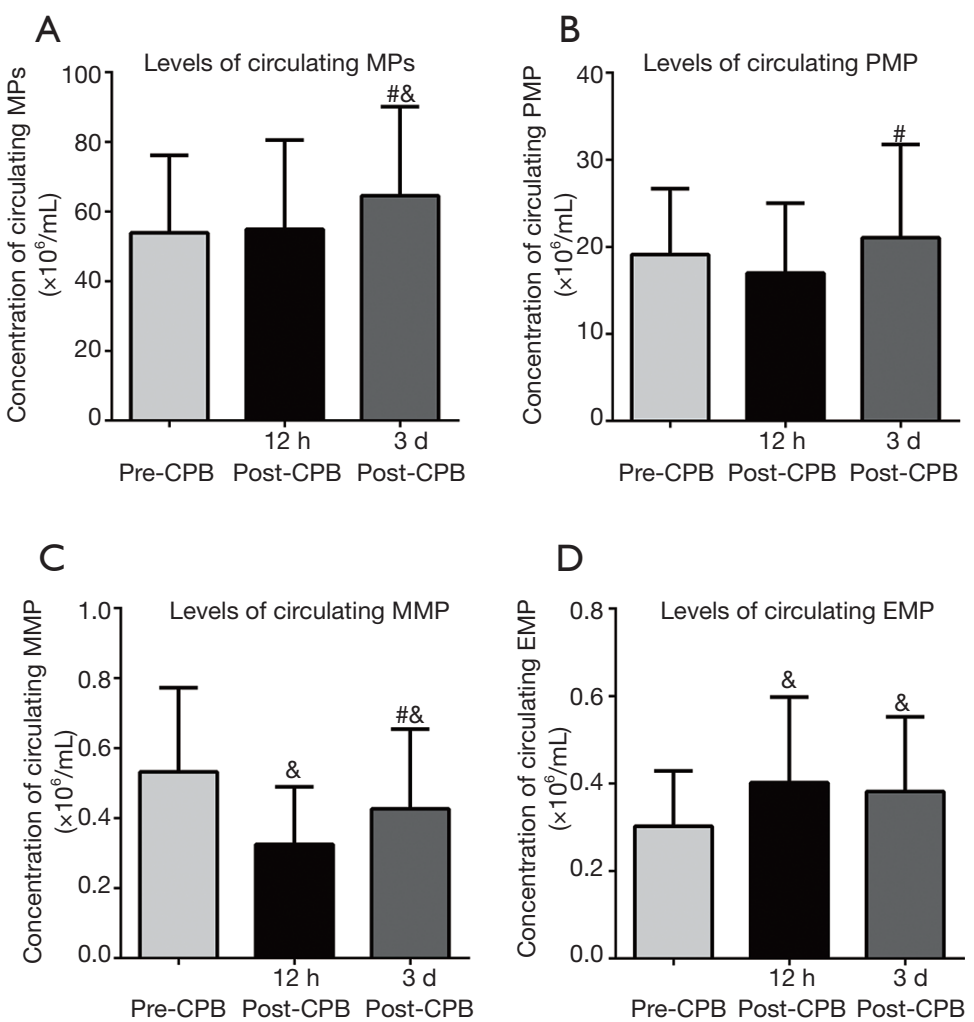

Figure 1 Cardiac surgery with CPB changes the levels of circulating MPs and its subpopulation. The plasma was isolated form enrolled patients at different times (per-operation, $12 \mathrm{~h}$ post-CPB, and $3 \mathrm{~d}$ post-CPB). The thawed plasma was incubated with different biomarkers to identify its source and detected by flow cytometry. Data were presented as mean \pm standard deviation (mean \pm SD); *, vs. pre-CPB; \#, vs. 12 h post-CPB, $\mathrm{P}<0.05$. CPB, cardiopulmonary bypass; MPs, circulating microparticles; PMP, platelet-derived microparticles; MMP, monocyte-derived microparticles; EMP, endothelial-derived microparticles.

seems to be less related with the types of cardiac surgery, and the degree of surgical complexity was associated with $\mathrm{AKI}$ at $3 \mathrm{~d}$ post-CPB (Table 1). As expected, the CPB time was increased in AKI both at $12 \mathrm{~h}$ post-CPB and $3 \mathrm{~d}$ post$\mathrm{CPB}$ groups than non-AKI groups, and similar changes were found in the operation time of cardiac surgery and cross clamp time (Table 1).

\section{The circulating MPs, especially EMP, elevated after CPB}

Previously, we demonstrated that the circulating MPs were increased in patients with VHD $(5,10)$. To explore the change of MPs and its subpopulations during the perioperative period of cardiac surgery, the association with $\mathrm{CPB}$ and FCM were performed to measure the levels of MPs and its subtypes.

The concentration of circulating MPs did not significantly change at $12 \mathrm{~h}$ post-CPB compared with
pre-CPB. However, MPs were elevated at $3 \mathrm{~d}$ post-CPB (Figure 1A). The PMP, most abundant MPs in circulation, showed similar changes with MPs, and were elevated at $3 \mathrm{~d}$ post-CPB (Figure 1B). The CPB significantly reduced the level of $\mathrm{MMP}$ at $12 \mathrm{~h}$ post-CPB compared with pre-CPB, and the MMP slightly returned at $3 \mathrm{~d}$ post-CPB, especially compared with $12 \mathrm{~h}$ post-CPB (Figure 1C). The EMP was significantly elevated at $12 \mathrm{~h}$ post-CPB, and their release was slightly lower at $3 \mathrm{~d}$ post-CPB compared with $12 \mathrm{~h}$ post-CPB, but still higher than that of preoperative values (Figure 1D).

\section{EMP elevated in AKI at 12 b post-CPB}

To further investigate the correlation of the subtypes of circulating MPs with AKI after cardiac surgery, the subpopulations of MPs were labeled with specific markers of source cells such as CD144 for EMP, CD41 for PMP, 
and CD14 for MMP. Figure 2 illustrates the changes in the concentration of the subpopulations of circulating MPs at three time points (pre-CPB, $12 \mathrm{~h}$ post-CPB, and $3 \mathrm{~d}$ post$\mathrm{CPB}$ ) in the $\mathrm{AKI}$ and non-AKI groups occurred at $12 \mathrm{~h}$ and 3 d post-CPB.

In patients with $\mathrm{AKI}$ at $12 \mathrm{~h}$ post-CPB (AKI-12 h), the concentration of $\mathrm{PMP}$ was not altered at pre-CPB but elevated at $12 \mathrm{~h}$ post-CPB (Figure $2 \mathrm{~A}$ ). Interestingly, in the patients with AKI it occurred at $3 \mathrm{~d}$ post-CPB (AKI-3 d). The concentration of $\mathrm{PMP}$ at pre-CPB was not changed, but increased at both $12 \mathrm{~h}$ and $3 \mathrm{~d}$ post-CPB compared with the non-AKI group (Figure $2 B$ ).

Compared with the non-AKI patients, no significant change of levels of MMP at two different time points (pre$\mathrm{CPB}$, and $12 \mathrm{~h}$ post-CPB) were detected in the patients with AKI occurred at $12 \mathrm{~h}$ post-CPB (Figure $2 \mathrm{C}$ ). There was also no difference in the MMP levels in the perioperative period between AKI at $3 \mathrm{~d}$ post-CPB and the non-AKI group (Figure 2D).

The EMP at pre-CPB showed no difference between AKI and non-AKI groups at $12 \mathrm{~h}$ or $3 \mathrm{~d}$ post-CPB (Figure 2E,F). Compared with the patients without AKI, the EMP levels at $12 \mathrm{~h}$ post- CPB showed a remarkable increase in the AKI group at $12 \mathrm{~h}$ post-CPB (Figure $2 \mathrm{E}$ ). Figure $2 F$ shows the concentration of EMP at $12 \mathrm{~h}$ and $3 \mathrm{~d}$ post-CPB elevated in the patients with $\mathrm{AKI}$ at $3 \mathrm{~d}$ post-CPB than in the non-AKI (Figure 2F).

\section{Circulating EMP as a potential risk factor in predicting if AKI occurred post $C P B$}

Table 2 describes the associated factors with AKI at $12 \mathrm{~h}$ and $3 \mathrm{~d}$ post-CPB. The univariate analysis for AKI at $12 \mathrm{~h}$ post-CPB illustrated that the operation time, $\mathrm{CPB}$ time, cross clamp time, and the levels of PMP and EMP at $12 \mathrm{~h}$ post-CPB led to the elevated risk of AKI at $12 \mathrm{~h}$ post-CPB (Table 2). Further multivariate logistic analysis indicated that the concentrations of EMP and PMP were independent risk factors for $\mathrm{AKI}$ at $12 \mathrm{~h}$ post-CPB, and the EMP was the most promising biomarker for AKI at $12 \mathrm{~h}$ post-CPB (Table 2).

To further explore the risk factors for AKI which occurred at $3 \mathrm{~d}$ post-CPB, the logistic analysis was performed and similar results to AKI at $12 \mathrm{~h}$ post-CPB (Table 2) were found. The univariate analysis found that DM, operation time, CPB time, cross clamp time, and EMP and $\mathrm{PMP}$ at $3 \mathrm{~d}$ post-CPB were the associated factors with AKI which occurred at $3 \mathrm{~d}$ post-CPB (Table 2). The next multivariate logistic analysis also showed that the EMP, not $\mathrm{PMP}$ at $3 \mathrm{~d}$ post-CPB correlated with AKI (Table 2).

\section{Diagnostic value of EMP for AKI occurred at post-CPB}

The ROC curve was performed to detect the diagnostic value of EMP for AKI at post-CPB. The AUC for the counts of $\mathrm{EMP}$ at $12 \mathrm{~h}$ post-CPB was 0.86 (Figure $3 A$ ). Table 3 shows that the best cut-off valve of EMP in diagnosing AKI at $12 \mathrm{~h}$ post-CPB was $3.99 \times 10^{5}$ particles $/ \mathrm{mL}$, and the sensitivity and specificity were 0.88 and 0.88 , respectively (Table 3 ).

To further evaluate the diagnostic value of EMP in AKI at $3 \mathrm{~d}$ post-CPB, the ROC curve analysis was conducted and the value of AUC was 0.91 (Figure 3B). The best cutoff value of EMP in diagnosing AKI at $3 \mathrm{~d}$ post-CPB was $4.56 \times 10^{5}$ particles $/ \mathrm{mL}$ with a sensitivity of 0.85 and a specificity of 0.91 (Table 3).

\section{Discussion}

We previously demonstrated that circulating MPs are increased in heart disease such as VHD (5) and CHD (8). It induces neutrophil chemotaxis in the kidneys (18), which may be the underlying cause of kidney injury associated with cardiac disease. Accumulating evidence proved that MPs and its subtypes including EMP are elevated in AKI and is becoming a novel biomarker of AKI $(4,15,22)$. In this study, we found that the circulating EMP were elevated in the patients undergoing cardiac surgery with $\mathrm{CPB}$, which further increased with the occurrence of AKI at both $12 \mathrm{~h}$ and $3 \mathrm{~d}$ post-CPB and was a promising diagnostic marker for CPB-AKI.

The KDIGO criteria are conducted to define CPBAKI mainly depending on the increase of serum creatinine ( $\geq 26.5 \mu \mathrm{mol} / \mathrm{L}$ within $48 \mathrm{~h}$ or $1.5-1.9$ times the baseline levels within 7 days) (20). Among the 85 patients enrolled, the incidence of CPB-AKI was $40 \%$ (12 h post-CPB) and $31.76 \%$ (3 d post-CPB), which are similar to other studies ranging from $5 \%$ to $42 \%$ (23). We found that the patientrelated factors such as old age, drinking, DM, and the operation-related factors including complex cardiac surgery, CPB time, cross clamp time, and operation time are the potential risk factors for AKI after CPB (24). Others also found that old age and female sex are associated with CPBAKI (25) and the different results from us maybe due to the small sample size in this study.

Although the serum creatinine is the gold standard to diagnose AKI in clinically, its use is limited due to the 

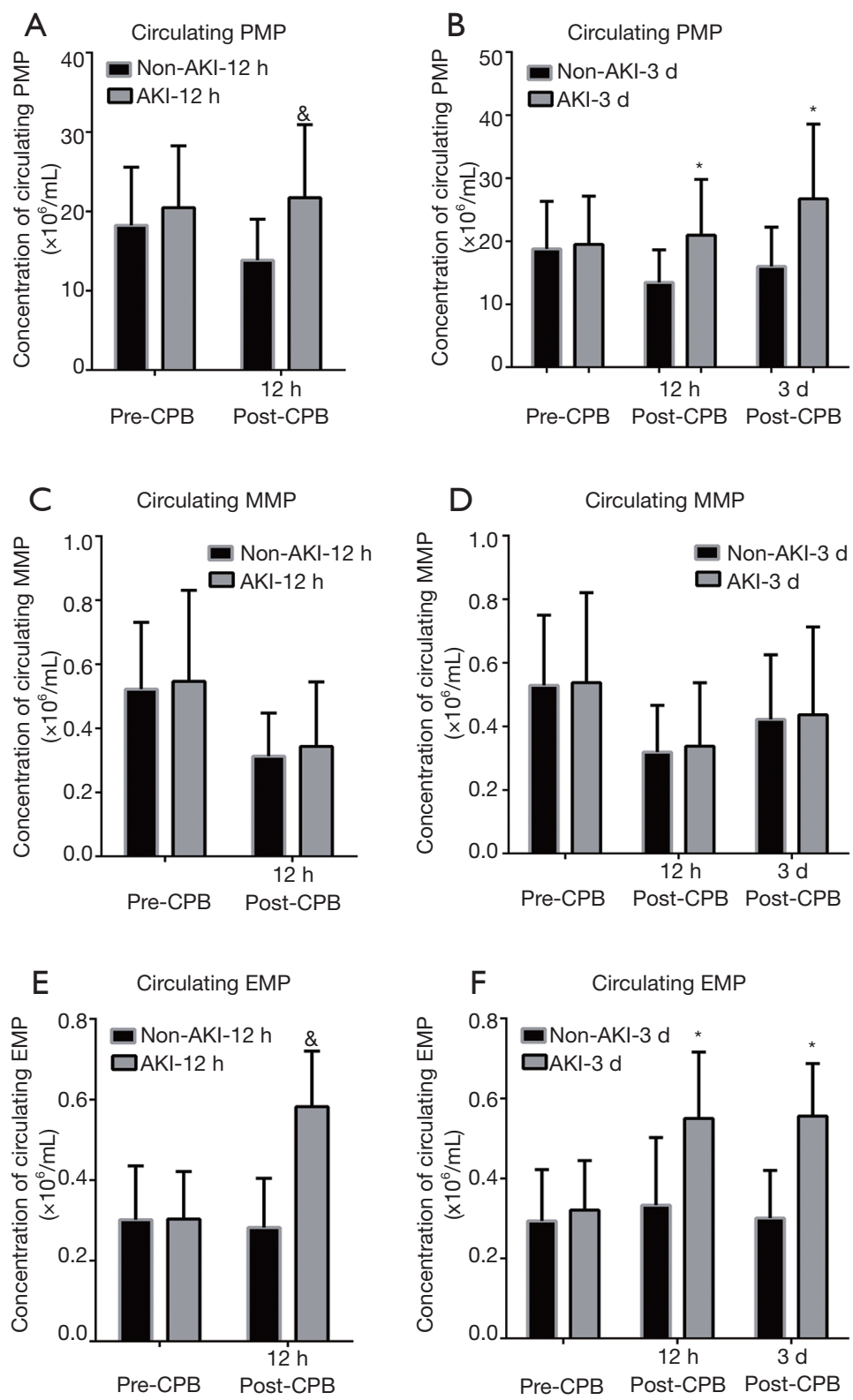

Figure 2 Changes in the concentrations of circulating EMP, MMP, and PMP in the AKI and non-AKI group post-CPB. The enrolled patients were divided into the AKI and non-AKI groups according to the KIDGO at $12 \mathrm{~h}$ and $3 \mathrm{~d}$ post-CPB and the levels of subtypes of circulating MPs (EMP, MMP, and PMP) detected by a flow cytometry were compared. (A) The bar chart shows the levels of circulating $\mathrm{PMP}$ in the patients with AKI group occurred at $12 \mathrm{~h}$ post-CPB and non-AKI group; (B) the bar chart shows the levels of circulating PMP in the patients with AKI group occurred at $3 \mathrm{~d}$ post-CPB and non-AKI group; (C,D) the bar chart described the changes of MMP in the $\mathrm{AKI}$ at $12 \mathrm{~h}(\mathrm{C})$ and $3 \mathrm{~d}(\mathrm{D})$ post-CPB and non-AKI; (E,F) the alteration of EMP in the patients undergoing cardiac surgery with or without $\mathrm{AKI}$ at $12 \mathrm{~h}(\mathrm{E})$ and $3 \mathrm{~d}(\mathrm{~F})$ post-CPB. Data were presented as mean \pm standard deviation (mean $\pm \mathrm{SD}) .{ }^{*}, v s$. non-AKI-12 h group; *, vs. non-AKI-3 d group, $\mathrm{P}<0.05$. CPB, cardiopulmonary bypass; PMP, platelet-derived microparticles; MMP, monocyte-derived microparticles; EMP, endothelial-derived microparticles. 
Table 2 Univariate and multivariate analysis of potential risk factors for AKI occurred after operation with CPB

\begin{tabular}{|c|c|c|c|c|}
\hline & \multicolumn{2}{|c|}{ Univariate } & \multicolumn{2}{|c|}{ Multivariate $\S$} \\
\hline \multicolumn{5}{|l|}{ Postoperative $12 \mathrm{~h}$} \\
\hline Age & $1.06(1.01-1.11)$ & 0.015 & & \\
\hline Gender & $0.57(0.15-1.26)$ & 0.213 & & \\
\hline Operation time & $1.01(1-1.01)$ & 0.001 & & \\
\hline CPB time & 1.02 (1.01-1.02) & $<0.001$ & No select & \\
\hline Cross clamp time & $1.02(1.01-1.03)$ & 0.001 & & \\
\hline PMP $-12 \mathrm{~h}^{\dagger}$ & $1.13(1.05-1.21)$ & 0.001 & $1.11(1.01-1.22)$ & 0.023 \\
\hline Age & $1.03(0.98-1.08)$ & 0.201 & & \\
\hline Gender & $1.06(0.42-2.65)$ & 0.908 & & \\
\hline Diabetes mellitus & $9.8(1.88-51.15)$ & 0.007 & & \\
\hline Operation time & $1(1.00-1.01)$ & $<0.001$ & & \\
\hline CPB time & $1.02(1.01-1.03)$ & $<0.001$ & No select & \\
\hline Cross clamp time & $1.02(1.01-1.03)$ & $<0.001$ & & \\
\hline PMP-3d ${ }^{\dagger}$ & $1.13(1.07-1.2)$ & $<0.001$ & $1.06(0.99-1.14)$ & 0.106 \\
\hline EMP $-3 d^{\ddagger}$ & $4.05(2.26-7.25)$ & $<0.001$ & 3.18 (1.69-5.97) & $<0.001$ \\
\hline
\end{tabular}

${ }^{\dagger}$, variable units is $\times 10^{6}$ particles $/ \mathrm{mL} .{ }^{\ddagger}$, variable units is $\times 10^{5}$ particles $/ \mathrm{mL}$. ${ }^{\S}$, adjust model adjust for: drinking and cross clamp time at postoperative $12 \mathrm{~h}$, operation time and cross clamp time at postoperative $3 \mathrm{~d}$. AKl, acute kidney injury; CPB, cardiopulmonary bypass. PMP-12h, platelet-derived microparticles at $12 \mathrm{~h}$ post-CPB; EMP-12h, endothelial-derived microparticles at $12 \mathrm{~h}$ post-CPB; PMP-3d, platelet-derived microparticles at $3 \mathrm{~d}$ post-CPB and EMP-3d, endothelial-derived microparticles at $3 \mathrm{~d}$ post-CPB. OR, odds ratio; Cl, confidence interval.

physiological processing, drugs, and diabetes which could affect the serum creatinine level (26). Some biomarkers for AKI such as kidney injury molecule-1, IL-18, and neutrophil gelatinase-associated lipocalin are investigated. However, large trials show a weaker diagnostic ability with AUC less than 0.77 (27). Therefore, new potential markers are needed to explore. We previously found that circulating MPs detected by FCM are increased in patients with cardiac disease compared with healthy participants. However, surgery did not alter the levels of MPs at $12 \mathrm{~h}$ post-CPB $(5,10)$. Based on the results of MPs detected by FCM, we confirmed that cardiac surgery with $\mathrm{CPB}$ does not change the concentration of MPs at $12 \mathrm{~h}$ post-CPB including the main subtype PMP. Others' ex vivo experiments also found the fresh blood challenged with the CPB circuit does not increase plasma levels of PMP (28). During $\mathrm{CPB}$, complement activation and inflammatory response are aggregated and contributes to the release of MPs as predicted. The conflict results may be due to adhesion of PMP to CPB circuit, hemodilution or mechanical cell salvage during CPB (29). Another reason may be the detective limit of FCM, which are unable to measure the MPs (mainly ranging 100-300 nm) less than $300 \mathrm{~nm}$ $(21,30)$. The MPs levels including PMP were increased at $3 \mathrm{~d}$ post-CPB compared with $12 \mathrm{~h}$ and the underlying reason may be the recovery of blood volume and the reduction of $\mathrm{PMP}$ clearance from $\mathrm{CPB}$ with the treatment in the intensive care unit within $3 \mathrm{~d}$ after operation $(5,10)$.

Elevated EMP is becoming a promising biomarker in many cardiorenal disease based on endothelial dysfunction 

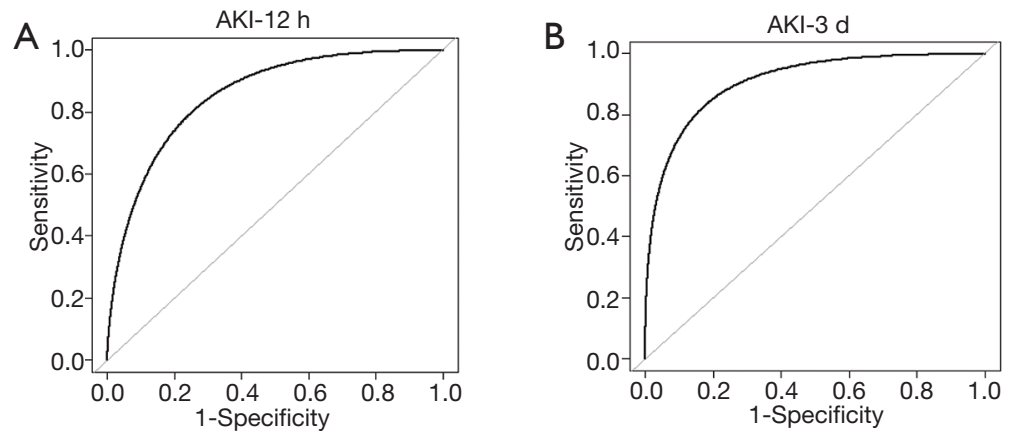

Figure 3 Receiver operating characteristic curve analysis of EMP levels. Receiver operating characteristic (ROC) curve analysis of EMP levels. AUC confidence interval and significance test using a nonparametric repeated sampling method (Bootstrap resampling times =500). (A) ROC for AKI occurred at $12 \mathrm{~h}$ post-CPB (AKI-12 h); (B) ROC for AKI at $3 \mathrm{~d}$ post-CPB (AKI-3d). EMP, endothelial-derived microparticles; AKI, acute kidney injury; CPB, cardiopulmonary bypass.

Table 3 Parameters of ROC curve for AKI happened after operation with CPB

\begin{tabular}{llccc} 
& & & & \multicolumn{2}{c}{ At cut-off value } \\
\cline { 4 - 5 } & AUC $(95 \% \mathrm{Cl})$ & Cut-off value $\left(\times 10^{5}\right.$ particles $\left./ \mathrm{mL}\right)$ & Sensitivity & Specificity \\
\hline EMP-12h & $0.86(0.77-0.96)$ & 3.99 & 0.88 & 0.88 \\
EMP-3d & $0.91(0.83-0.97)$ & 4.56 & 0.85 & 0.91 \\
\hline
\end{tabular}

ROC, receiver operating characteristic; AKI, acute kidney injury; CPB, cardiopulmonary bypass; AUC, area under the curve; EMP-12h, endothelial-derived microparticles at $12 \mathrm{~h}$ post-CPB; EMP-3 d, endothelial-derived microparticles at $3 \mathrm{~d}$ post-CPB.

such as CHD (8), VHD (7), atherosclerosis (9), DM (31), acute coronary syndrome (31), kidney failure (32), and endstage renal disease (33). Previously, we demonstrated that EMP isolated from endothelial cells aggravates endothelial dysfunction, leading to the anti-angiogenesis in the heart and acute lung injury $(6-8,16)$. In this study, we found that the level of EMP was elevated both at $12 \mathrm{~h}$ and $3 \mathrm{~d}$ after surgery with $\mathrm{CPB}$. The endothelium activation and injury increased the release of EMP, and endothelial cells mostly located at the surface of blood vessels will not be cleared during CPB. The endothelial injury induced by $\mathrm{CPB}$ mediates essential pathophysiological processes including the regulation of blood flow, inflammation, and vascular permeability in the AKI (34). The characteristic of reflecting endothelial injury makes the elevated EMP a specific biomarker of AKI possible.

Further investigation on the relationship between MPs including its subtypes and CPB-AKI found that the patients with AKI occurred at $12 \mathrm{~h}$ post-CPB meanwhile 3 d post-CPB shows high levels of PMP and EMP, not MMP. The next univariate logistic analysis found that both EMP and PMP are the risk factors for AKI at $12 \mathrm{~h}$ and $3 \mathrm{~d}$ post-CPB. However, the multivariate logistic analysis revealed that EMP was a more powerful diagnostic biomarker for CPB-AKI. The association between MPs and CPB-AKI are rarely reported. To the best of our knowledge, it is the first time to perform an accurate count of EMP measured by FCM to diagnose AKI after cardiac surgery. We found that the best cut-off valves of EMP are $3.99 \times 10^{5}$ and $4.56 \times 10^{5}$ particles $/ \mathrm{mL}$, with the specificity of 0.88 and 0.91 for $12 \mathrm{~h}$ and $3 \mathrm{~d}$ after surgery, respectively. Some studies found the EMP are elevated in the cardiorenal dysfunction-related diseases. In the patients with acute myocardial infraction, EMP are further increased with renal dysfunction (35). EMP are also elevated in CKD patients with coronary artery disease compared with non-coronary artery disease (36). These findings may provide a promising biomarker of EMP for the early diagnosis of CPB-AKI. These findings are very important since $12 \mathrm{~h}$ post-CPB is an early stage after cardiac surgery, and the doctors may prevent the occurrence of AKI or treat the patients at an earlier stage of renal function failure through detecting this biomarker clinically.

Previously, we found that the circulating MPs, especially EMP, are increased in heart disease $(7,8)$ and the elevated MPs induced the neutrophil accumulated in 
the kidneys (18). EMP were derived from endothelial cells upon activation and other stimulations. The glomerulus endothelial dysfunction is the initial step of AKI (34). The changes of EMP reflect the conditions of endothelial function and glomerulus endothelial function $(8,19,29,37)$. The elevated EMP after cardiac surgery can induce endothelial dysfunction and aggravate kidney injury. Thus, EMP may be used to early predict AKI occurrence with more specificity and sensitivity after cardiac surgery.

Several limitations exist in this study. First, these results are based on the measurement of EMP performed on FCM; the widely used technique to determine the cellular origin of individual MPs and the concentration of MPs. The electron microscope confirms that the size of MPs ranges from 100 to $1,000 \mathrm{~nm}(10,21,30)$. Due to the technical factors of FCM, parts of MPs are missed. To reduce error, we used the Megamix-Plus SSC beads with four diameters (160, 200, 240, and $500 \mathrm{~nm}$ ) to quantify the MPs. Second, the heart valve replacement surgery is the major surgery in this study, which needs CPB. Only few cases of cardiac surgery were performed without $\mathrm{CPB}$. Therefore, the number of cases was insufficient as a control variable for patients undergoing cardiac surgery without CPB. Future study will use circulating microparticle levels on patients undergoing cardiac surgery without $\mathrm{CPB}$ as a control. Other limitations are the small sample size and the heterogeneity with respect to other modality of operation.

\section{Conclusions}

In summary, our present study shows the diagnostic value of EMP for AKI occurred after cardiac surgery with CPB. We found that the concentration of EMP detected by FCM could predict $\mathrm{CPB}-\mathrm{AKI}$ at $12 \mathrm{~h}$ and $3 \mathrm{~d}$ post-CPB with high specificities.

\section{Acknowledgments}

The authors would like to thank the patients as well as staffs at the First Affiliated Hospital, Sun Yat-sen University for their assistance throughout this study. The authors also thank the editage for the editing of the language.

Funding: This work was supported by the National Natural Science Foundation of China (Grants 81770241, 81830013, 82000362, 81670392, 81600382, 81970363), the Changjiang Scholars Program from the Ministry of Education of China, Guangdong Natural Science Fund Committee (Grant 2015A030312009), Guangdong Basic and Applied
Basic Research Foundation (Grant 2019B1515120092), the Sun Yat-sen University Clinical Research 5010 Program (2014002) and Program of National Key Clinical Specialties. Fundamental Research Funds for the Central Universities Sun Yat-sen University Young Teacher Training Program (19ykpy79).

\section{Footnote}

Reporting Checklist: The authors have completed the STARD reporting checklist. Available at http://dx.doi.org/10.21037/ atm-20-7828

Data Sharing Statement: Available at http://dx.doi. org/10.21037/atm-20-7828

Conflicts of Interest: All authors have completed the ICMJE uniform disclosure form (available at http://dx.doi. org/10.21037/atm-20-7828). The authors have no conflicts of interest to declare.

Ethical Statement: The authors are accountable for all aspects of the work in ensuring that questions related to the accuracy or integrity of any part of the work are appropriately investigated and resolved. This study complied with the declaration of Helsinki (as revised in 2013) and was approved by the Ethics Review Board of the First Affiliated Hospital, Sun Yat-sen University [(2011)14]. All subjects recruited in this study signed the prior written informed consent.

Open Access Statement: This is an Open Access article distributed in accordance with the Creative Commons Attribution-NonCommercial-NoDerivs 4.0 International License (CC BY-NC-ND 4.0), which permits the noncommercial replication and distribution of the article with the strict proviso that no changes or edits are made and the original work is properly cited (including links to both the formal publication through the relevant DOI and the license). See: https://creativecommons.org/licenses/by-nc-nd/4.0/.

\section{References}

1. Lagny MG, Jouret F, Koch JN, et al. Incidence and outcomes of acute kidney injury after cardiac surgery using either criteria of the RIFLE classification. BMC Nephrol 2015;16:76.

2. Qian Y, Che L, Yan Y, et al. Urine klotho is a potential 
early biomarker for acute kidney injury and associated with poor renal outcome after cardiac surgery. BMC Nephrol 2019;20:268.

3. Krawczeski CD. Cardiopulmonary Bypass and AKI: AKI Is Bad, So Let's Get Beyond the Diagnosis. Front Pediatr 2019;7:492.

4. Erdbrügger U, Le TH. Extracellular Vesicles in Renal Diseases: More than Novel Biomarkers? J Am Soc Nephrol 2016;27:12-26.

5. Fu L, Hu XX, Lin ZB, et al. Circulating microparticles from patients with valvular heart disease and cardiac surgery inhibit endothelium-dependent vasodilation. J Thorac Cardiovasc Surg 2015;150:666-72.

6. Ou ZJ, Chang FJ, Luo D, et al. Endothelium-derived microparticles inhibit angiogenesis in the heart and enhance the inhibitory effects of hypercholesterolemia on angiogenesis. Am J Physiol Endocrinol Metab 2011;300:E661-8.

7. Ci HB, Ou ZJ, Chang FJ, et al. Endothelial microparticles increase in mitral valve disease and impair mitral valve endothelial function. Am J Physiol Endocrinol Metab 2013;304:E695-702.

8. Lin ZB, Ci HB, Li Y, et al. Endothelial microparticles are increased in congenital heart diseases and contribute to endothelial dysfunction. J Transl Med 2017;15:4.

9. Chen YT, Yuan HX, Ou ZJ, et al. Microparticles (Exosomes) and Atherosclerosis. Curr Atheroscler Rep 2020;22:23.

10. Li Y, Yuan H, Chen C, et al. Concentration of circulating microparticles: a new biomarker of acute heart failure after cardiac surgery with cardiopulmonary bypass. Sci China Life Sci 2021;64:107-16.

11. Burger D, Schock S, Thompson CS, et al. Microparticles: biomarkers and beyond. Clin Sci (Lond) 2013;124:423-41.

12. Dursun I, Yel S, Unsur E. Dynamics of circulating microparticles in chronic kidney disease and transplantation: Is it really reliable marker? World J Transplant 2015;5:267-75.

13. Poon KS, Palanisamy K, Chang SS, et al. Plasma exosomal miR-223 expression regulates inflammatory responses during cardiac surgery with cardiopulmonary bypass. Sci Rep 2017;7:10807.

14. Chen YJ, Huang CS, Wang F, et al. Effect of ligustrazine hydrochloride on coagulation reaction and inflammation reaction in single valve replacement patients with rheumatic heart disease undergoing cardiopulmonary bypass. Zhongguo Zhong Xi Yi Jie He Za Zhi 2014;34:531-5.
15. Karpman D, Ståhl AL, Arvidsson I. Extracellular vesicles in renal disease. Nat Rev Nephrol 2017;13:545-62.

16. Densmore JC, Signorino PR, Ou J, et al. Endotheliumderived microparticles induce endothelial dysfunction and acute lung injury. Shock 2006;26:464-71.

17. Jian YP, Yuan HX, Hu KH, et al. Protein Compositions Changes of Circulating Microparticles in Patients With Valvular Heart Disease Subjected to Cardiac Surgery Contribute to Systemic Inflammatory Response and Disorder of Coagulation. Shock 2019;52:487-96.

18. Yuan HX, Chen CY, Li YQ, et al. Circulating extracellular vesicles from patients with valvular heart disease induce neutrophil chemotaxis via FOXO3a and the inhibiting role of dexmedetomidine. Am J Physiol Endocrinol Metab 2020;319:E217-31.

19. Tökés-Füzesi M, Woth G, Ernyey B, et al. Microparticles and acute renal dysfunction in septic patients. J Crit Care 2013;28:141-7.

20. Khwaja A. KDIGO clinical practice guidelines for acute kidney injury. Nephron Clin Pract 2012;120:c179-84.

21. Royo F, Thery C, Falcon-Perez JM, et al. Methods for Separation and Characterization of Extracellular Vesicles: Results of a Worldwide Survey Performed by the ISEV Rigor and Standardization Subcommittee. Cells 2020;9:1955.

22. Zhang W, Zhou X, Zhang H, et al. Extracellular vesicles in diagnosis and therapy of kidney diseases. Am J Physiol Renal Physiol 2016;311:F844-51.

23. Wang Y, Bellomo R. Cardiac surgery-associated acute kidney injury: risk factors, pathophysiology and treatment. Nat Rev Nephrol 2017;13:697-711.

24. Coleman MD, Shaefi S, Sladen RN. Preventing acute kidney injury after cardiac surgery. Curr Opin Anaesthesiol 2011;24:70-6.

25. O'Neal JB, Shaw AD, Billings FTt. Acute kidney injury following cardiac surgery: current understanding and future directions. Crit Care 2016;20:187.

26. Samra M, Abcar AC. False estimates of elevated creatinine. Perm J 2012;16:51-2.

27. Siew ED, Ware LB, Ikizler TA. Biological markers of acute kidney injury. J Am Soc Nephrol 2011;22:810-20.

28. Atherton J, Hampshire T, Englyst N, et al. The effect of circulating fresh blood through a micro-bypass circuit on platelet microparticles. Anaesthesia 2012;67:555.

29. Tempo JA, Englyst NA, Holloway JA, et al. Platelet Microvesicles (Microparticles) in Cardiac Surgery. J Cardiothorac Vasc Anesth 2016;30:222-8.

30. Konokhova AI, Chernova DN, Strokotov DI, et al. 
Page 12 of 12

Light-scattering gating and characterization of plasma microparticles. J Biomed Opt 2016;21:115003.

31. Koga H, Sugiyama S, Kugiyama K, et al. Elevated levels of VE-cadherin-positive endothelial microparticles in patients with type 2 diabetes mellitus and coronary artery disease. J Am Coll Cardiol 2005;45:1622-30.

32. Dursun I, Poyrazoglu HM, Gunduz Z, et al. The relationship between circulating endothelial microparticles and arterial stiffness and atherosclerosis in children with chronic kidney disease. Nephrol Dial Transplant 2009;24:2511-8.

33. Boulanger CM, Amabile N, Guérin AP, et al. In vivo shear stress determines circulating levels of endothelial microparticles in end-stage renal disease. Hypertension 2007;49:902-8.

Cite this article as: Ma J, Yuan HX, Chen YT, Ning DS, Liu XJ, Peng YM, Chen C, Song YK, Jian YP, Li Y, Liu Z, Ou ZJ, Ou JS. Circulating endothelial microparticles: a promising biomarker of acute kidney injury after cardiac surgery with cardiopulmonary bypass. Ann Transl Med 2021;9(9):786. doi: $10.21037 /$ atm-20-7828

\section{Ma et al. Circulating MPs predict AKI after cardiac surgery}

34. Verma SK, Molitoris BA. Renal endothelial injury and microvascular dysfunction in acute kidney injury. Semin Nephrol 2015;35:96-107.

35. Mörtberg J, Lundwall K, Mobarrez F, et al. Increased concentrations of platelet- and endothelial-derived microparticles in patients with myocardial infarction and reduced renal function- a descriptive study. BMC Nephrol 2019;20:71.

36. Chen YL, Chen CH, Wallace CG, et al. Levels of Circulating Microparticles in Patients with Chronic Cardiorenal Disease. J Atheroscler Thromb 2015;22:247-56.

37. Voukalis C, Shantsila E, Lip GYH. Microparticles and cardiovascular diseases. Ann Med 2019;51:193-223. 